(Aus dem physiologischen Institut der Universität Bonn.)

\title{
Leitungsverlangsamung und Verringerung des Stoffumsatzes als Grundlage der scheinbaren ,Gewöhnung“6 des wärmegelähmt gewesenen Nerven.
}

\author{
Von \\ Dr. med. Walter Thörner, \\ Priv.-Doz. an der Universität Bonn. \\ (Eingegangen am 1. Mai 1922)
}

Erwärmt man einen Abschnitt des Nerven eines Nervmuskelpräparates in indifferentem Medium, so erlischt bekanntlich bei einer bestimmten Temperatur, deren Höhe von verschiedenen Faktoren - Froschart undZustand, Länge der Strecke, Geschwindigkeit des Temperaturanstiegs, Sauerstoffgehalt des Mediums - abhängt, das Leitvermögen, $d . \mathbf{h}$. die erwärmte Nervenstrecke vermag zentral von ihr angebrachte Erregungen nicht mehr hindurchzulassen, sie ist wärmegelähmt. Geringe Abkühlung bringt das Leitvermögen zurück und zwar vollständig, soweit das an der Größe der Muskelzuckung gemessen werden kann. Wiederholt man nun aber die Erwärmung unter gleichen Bedingungen, so verschwindet das Leitvermögen jetzt bei einem höheren Temperaturgrade als bei der ersten Erwärmung und zwar ist diese Zunahme der Widerstandsfähigkeit des Nerven gegen die Wärmewirkung um so größer, je stärker und länger innerhalb gewisser Grenzen die Übererwärmung über den Temperaturgrad des ersten Leitfähigkeitsverlustes vorgenommen war. Diese Beobachtungen sind als scheinbare ,Gewöhnung" bezeichnet und samt den Bedingungen, unter denen sie zustande kommen, a. a. O.1) ausfürlich behandelt.

Hier diene zur Erläuterung dieser Erscheinungen eine kurze zahlenmäBige Zusammenstellung der damaligen Ergebnisse, wobei aber wegen des Vergleiches mit der vorliegenden Untersuchung nur die Versuche an Winterexemplaren von rana temporaria berücksichtigt seien (rana esculenta verliert das Nervenleitvermögen erst bei einer um $6-8^{\circ}$ höheren Temperatur und bei Sommerfröschen liegt die Wärmelähmungstem-

1) Thörner, Wärmeerregung, Wärmelähmung und der Erscheinungskomplex der ,Gewöhnung“ bei der letzteren. Zeitschr. f. allgem. Physiol. 18, 1919, Heft 2, S. 226 f. 
peratur um ea. $2^{\circ}$ niedriger). Ferner sei aus demselben Grunde nur auf die Gruppe der damaligen Versuche Bezug genommen, die mit gleichmäßigem Temperaturanstieg von $1-2^{\circ}$ pro 1 Min. an einer beeinflußten Strecke von $3 \mathrm{~cm}$ ausgeführt waren, da besonders bei Kaltfröschen ein langsamerer Temperaturanstieg infolge „Gewöhnung“ leicht zu einer erhöhten Wärmelähmungstemperatur führt und diese andrerseits um so höher liegt, je kürzer die erwärmte Strecke ist (ein Ausdruck des dekrementiellen Erregungsablaufes in derselben).

Unter den angenebenen Bedingungen stellten wir damals fest, daß der Nerv (N. ischiadicus) bei durchschnittlich $35^{\circ} \mathrm{C}$ seine Leitfähigkeit verlor (Sommertemporarien bei $33^{\circ} \mathrm{C}$, Eskulenten bei $42^{\circ} \mathrm{C}$ ). Ließ man die Temperatur nur wenig weiter ansteigen, etwa auf $36^{\circ}$, kühlte dann schnell ab, etwa bis auf $18^{\circ}$, was zu voller Erholung führte, und erwärmte den Nerven aufs neue, so büßte er sein Leitvermögen bei wenig erhöhter Temperatur, etwa bei $35^{\circ}$ ein. Trieb man jedoch die Erhitzung stark über den ersten Wärmelähmungsgrad hinaus, etwa bis $40^{\circ}$, so erfolgte bei einer abermaligen Erwärmung des inzwischen durch Abkühlung erholten Nerven die neue Lähmung erst bei einer um mehrere Grade, im Höchstfall am $4^{\circ}$, erhöhten Temperatur, also etwa bei $38-39^{\circ}$. Auch nach so starken Übererwärmungen erholte sich der Nerv vollständig, nach Maßgabe der Muskelzuckung. Nach noch höheren Temperaturen ließ diese aber an Größe nach, als Zeichen eines irreversiblen Ausfalls einer Anzahl von Nervenfasern in der behandelten Strecke. Mit Überschreiten der $40^{\circ}$ betreten wir das Gebiet der Dauerschädigungen, hier liegt für den Temporarianerven die Todestemperatur, gegenüber der Wärmelähmungstemperatur bei $34-35^{\circ}$.

Von $34^{\circ}$ bis $40^{\circ}$ aber erstreckt sich innerhalb bestimmter Zeitgrenzen das Reich der reversiblen Schädigungen mit dem wir uns im folgenden wesentlich zu beschäftigen haben. Die hier durch die Wärme bedingten Störungen bilden sich bei erniedrigter Temperatur zurück, falls sie nicht zu lange bestanden haben. Zur Deutung der in diesem Temperaturbereich beobachteten ,Gewöhnungs "-Erscheinungen hatten wir ${ }^{1}$ ) als solche reversible Schädigungen vor allem zwei herangezogen: Erstens eine funktionelle Störung im Stoffwechsel, die in dem Überwiegen des Sauerstoffbedarfs zufolge der gesteigerten Lebensprozesse über die mögliche Zufuhr, also in relativem Sauerstoffmangel, besteht und als Erstickung den Hauptfaktor für das Zustandekommen der Wärmelähmung ausmacht. Und zweitens eine beginnende Gerinnung, eine allmähliche Teilchenvergrößerung kolloidaler Protoplasmastoffe, die zunächst reversibel bleibend $\mathrm{zu}$ einer Stoffwechselverlangsamung führt und mit der Temperatur zunehmend erst in höheren Graden zu

1) Thörner. a. a. O. S. 271. 
gröberer Ausflockung und damit zum Wärmetode hinüberleitet. Also fließende Übergänge aus dem Bereich der Wärmelähmung in das des Wärmetodes; vermutlich wird durch die Erstickungsprodukte der Ausflockungsvorgang noch befördert, wodurch der Wärmetod auch bei den niedrigeren Temperaturen der Wärmelähmung erklärt wird, wenn sie nur lange genug unterhalten werden.

Eben die zweite der reversiblen Schädigungen, die beginnende $\mathrm{Zu}$ standsänderung in den kolloidalen Lösungsverhältnissen des Protoplasmas, erschien uns geeignet zur Erklärung der ,Gewöhnung“, indem wir der Meinung waren, daß die Verschlechterung der Lösungsbedingungen im Protoplasma eine Erschwerung der Stoffwechselvorgänge zur Folge habe und daß der durch diese Stoffwechselverlangsamung gegebene geringere Sauerstoffbedarf den Nerven widerstandsfähiger gegen die Erstickungsgefahr der Wärmewirkung mache, also gerade gegen jene erste Wärmeschädigung, auf der die Wärmelähmung beruht. Es arbeitet also gewissermaßen dieser beginnende Gerinnungsvorgang als stoffwechselherabsetzend, der die Umsetzungen steigernden Wärmewirkung entgegen.

So erklärt es sich, daß bei langsam steigender Temperatur $\left(1^{\circ}\right.$ in 2-3 Min.) die Lähmungstemperatur hinaufgeschoben werden kann. Natürlich müssen diese ersten Gerinnungsstufen immerhin einen gewissen Grad und Umfang erreicht haben, durch genügend lange Einwirkung möglichst hoher (in den angegebenen Grenzen) Temperaturen, damit die Stoff wechselverlangsamung den Sauerstöffbedarf soweit einschränke, daß es nach außen bemerkbar wird; so verstehen wir, daß nach starken Übererwärmungen bei sehr langsamer Abkühlung die Leitfähigkeit schon wiederkehrt bei Temperaturen, die $1-2^{\circ}$ höher liegen als die, bei denen sie im Aufstieg verschwunden war, und daß der Umfang der Gewöhnung um so größer ist, je höher die Übererwärmung war. Und schließlich ist zu fordern, daß im Gegensatz zu der funktionellen Störung der Erstickung die durch die Wärme bedingte kolloidale Zustandsänderung auch nach der Abkühlung bestehen bleibe, wenn sie bei einer erneuten Erwärmung irgendwie zum Ausdruck kommen soll, eine Forderung, deren Erfüllung durchaus im Wesen derartiger Vorgänge liegt. Und dennoch müssen wir diese ersten Gerinnungsstadien, wenn wir sie der „Gewöhnung“" zugrunde legen wollen, im Gegensatz zu den zum Wärmetod führenden höheren irreversiblen Graden als reversibel bezeichnen, weil wir sehen, daß die durch eine starke Übererwärmung gewonnene erhöhte Widerstandsfähigkeit gegen erneute Erwärmung allmählich zurückgeht, wenn wir das Präparat einige Stunden bei Zimmertemperatur ruhig liegen lassen. So zeigt z. B. ein Nerv, der bei der zweiten Wärmelähmung eine ,Gewöhnung" um $3,5^{\circ}$ aufwies, bei einer dritten Erwärmung, die nach fünfstündigem Liegen bei $18^{\circ}$ vorgenommen 
wird, nur noch eine ,Gewöhnung" um $1^{\circ}$ gegenüber der ersten Wärmelähmungstemperatur. Wenn aber die „Gewöhnung“ zurückgeht, müssen auch die sie bedingenden Momente rückbildungsfähig sein. Und sie müssen das auch aus dem Grunde, daß wir sie ja sonst den irreparablen Dauerschädigungen zurechnen müßten, während wir doch sehen, daß ein wärmegelähmt gewesener Nerv nicht nur viele Stunden funktionsfähig bleibt, sondern, wie im folgenden gezeigt werden soll, an Funktionstüchtigkeit wieder zunimmt.

Wenn wir nun das Wesen der Wärmelähmung in einer Erstickung sehen und andererseits die nach starken Erwärmungen beobachtete scheinbare ,Gewöhnung" an höhere Temperaturen zurückführen auf eine durch kolloidale Zustandsänderungen bedingte Stoffwechselverlangsamung, die den Sauerstoffbedarf des Nerven einschränkt, so ist zu fordern, daß ein wärmegelähmt gewesener ,gewöhnter" Nerv auch widerstandsfähiger gegen Sauerstoffmangel bei Zimmertemperatur sei und z. B. ein Verweilen in reinem Stickstoff länger aushalte, ohne Erregbarkeit und Leitvermögen zu verlieren, als ein nicht vorbehandelter Vergleichsnerv. Über solche Versuche ist bereits berichtet worden ${ }^{1}$ ). Es hat sich tatsächlich ergeben (an Sommerfroschnerven), daß ein ,gewöhnter" Nerv in reinem Stickstoff der Erstickung länger trotzt als ein normaler Nerv und zwar um ca. 8\% der Erstickungszeit des letzten. Auch an Winterfröschen werden neuerdings gleichgerichtete Versuche angestellt, die ebenfalls, wenn auch mit größeren Schwankungen, ergaben, daß der ,gewöhnte" Nerv die größere Widerstandskraft gegen Sauerstoffentziehung besitzt. Erschwerend machen sich allerdings bei den Kaltfröschen die sehr langen Erstickungszeiten, 4-5 Std., bemerkbar, da während dieser langen Zeit sich die Gewöhnungsbedingungen z.T. zurückbilden und somit dem gewünschten Resultat entgegengewirkt wird. Zudem kommen mit Zunahme der Erstickungsdauern individuelle Verschiedenheiten im Sauerstoffbedarf der Vergleichsnerven stärker zur Geltung und verdecken leicht den kleinen Zeitzuwachs des ,gewöhnten" Nerven.

Um das Zustandekommen des Erscheinungskomplexes ảer ,Gewöhnung" weiter aufzuhellen und die gegebene Deutung zu stützen, wurde versucht, die geforderte Stoffwechselverlangsamung in anderer Weise zu demonstrieren. Der Nerv, und speziell der markhaltige, ist diejenige Form der lebendigen Substanz, die am längsten allen Versuchen, durch Veranlassung zu dauernder Arbeitsleistung Ermüdungssymptome an ihm hervorzurufen, getrotzt hat. Seine geringe Ermüdbarkeit beruht auf der außerordentlichen Kürze seines Refraktärstadiums, das absolut nur etwa 0,001 und relativ 0,1 Sek. beträgt (Temporaria). Das heißt

1) Thörner, a. a. O. S. 264. 
aber nichts anderes als daß in dieser kurzen Zeit bereits alle Spuren einer Erregung an der betroffenen Stelle geschwunden sind und sie wieder voll funktionsfähig ist. Wenn wir den Erregungsvorgang als eine Stoffwechselschwankung ansehen, die in Zerfall und Wiederaufbau besteht, so ist also der Nerv mit einem außerordentlich geschwind ablaufenden Stoffumsatz (Funktionsstoffwechsel) begabt. Und eben darauf beruht auch die große Schnelligkeit, mit der der Nerv eine Erregung über seine ganze Länge fortpflanzt und in der er alle andern Formen der lebendigen Substanz übertrifft. Erst als man Methoden anwandte, durch die das Refraktärstadium in die Länge gezogen, d. h. der Stoffwechsel verlangsamt wurde - Abkühlung, Erstickung - gelang eine Ermüdung durch Dauerreizung ohne weiteres. Gleichzeitig ließ sich durch dieselben Mittel eine Abnahme der Leitungsgeschwindigkeit erzielen. Wir wissen somit, daß eine Verlangsamung des Nervenstoffwechsels $u$. a. in einer A bnahme der Leitungsgesch windigkeit zum Ausdruck kommt und können diese Erfahrung für unsere Frage herbeiziehen, die wir nun folgendermaßen formulieren würden: Läßt sich am Nerven nach einer starken Wärmelähmung gleichzeitig mit der ,Gewöhnung" eine auch nach völliger Erholung zurückbleibende Leitungsverlangsamung nachweisen, die nach mehrstündiger Ruhe bei Zimmertemperatur zugleich mit der Gewöhnung wieder zurïckgeht?

\section{Einige Bemerkungen zur Methodik.}

Die Versuche, die der Beantwortung der gestellten Frage galten, wurden in den Monaten Januar-März 1922 an kühlgehaltenen Kellerfröschen (Temporaria) ausgeführt.

Der Nervus ischiadicus eines Gastrocnemiuspräparates wurde duroh eine a. a. ${ }^{1}{ }^{1)}$ genauer beschriebene doppelwandige Glaskammer gezogen, so daß eine $3,5 \mathrm{~cm}$ lange Nervenstrecke durch Umspülung mit warmer Kochsalzlösung in fein abstufbarer Weise erwärmt werden konnte. Außerhalb der Wärmekammer lagen, gegen Miterwärmung und Austrocknung durch besondere feucht gehaltene Gehäuse geschützt, oberhalb und unterhalb und in gleichen Abständen von der erwärmbaren Strecke die Nervenabschnitte, an denen durch die Platinelektrodenpaare o (central) und $\mathrm{p}$ (peripher) der elektrische Reiz (etwas übermaximaler Öffnungsinduktionsschlag) zur Bestimmung der Latenszeiten angebracht wurde. Die Elektrode $c$ diente zugleich für den Prüfungsreiz bei der Wärmelähmung. Der Polabstand jeder Einzelelektrode betrug bei c $2,5 \mathrm{~mm}$, bei $\mathrm{p} 2 \mathrm{~mm}$. Die Nervenstrecke $\mathrm{zwischen}$ den beiden Kathoden, den für die Bestimmung in Betracht kommenden Ausgangspunkten der Erregung maß 4,1 cm, von denen etwa 3,2 cm der Wärmewirkung unterworfen wurden. Der Reizstrom wurde von einem einfachen kleinen Schlitteninduktorium mit schwachen Wickelungen und Eisenkern geliefert, dem der primäre Strom aus einem Akkumulator durch Widerstand gedrosselt zufloß. Die Reizschwelle lag i. D. für c bei $260 \mathrm{~mm}$, für $\mathrm{p}$ bei $220 \mathrm{mmR}$. A. (Rollenabstand). Dementsprechend wurde für den etwas übermaximalen Reiz zur Latenzzeitbestimmung

1) Thörner, a. a. O. S. 245. 
ein R.A. für c von ca. $200 \mathrm{~mm}$ und für $\mathrm{p}$ von $190 \mathrm{~mm}$ angewandt. Die Stromschleifengrenze lag für $\mathrm{p}$ unterhalb $40 \mathrm{~mm}$ R.A.

Vom unverletzten Gastrocnemius, der in feuchter Kammer bei konstanter Zimmertemperatur gehalten wurde, erfolgte durch unpolarisierbare Elektroden die Ableitung der Aktionsströme zum Saitengalvanometer von Einthoven. Es hatte sich herausgestellt, daß die Aktionsströme bei Ableitung von der Oberfläche des intakten Muskels gleichmäßiger bleiben und mit der Versuchsdauer viel langsamer an Intensität abnehmen als die anfänglich vielleicht kräftigeren bei QuerschnittLängsschnittableitung. Die Schwankung der mäßig gespannten Saite wurde mit der Gurtenschen Registriervorrichtung auf Papierfilm bei großer Umdrehungsgeschwindigkeit aufgenommen. Der Reizmoment wurde durch den Kontaktapparat (von Garten) mit 0,7 o Verspätung (kontrolliert durch Einbruch des verstärkten Reizstromes in den Galvanometerkreis) markiert. Diese Zahl wäre daher den Latenzzeiten der Protokolle hinzuzurechnen, um ihre wirkliche Größe zu erhalten, die jedoch für unsere Betrachtungen nicht in Frage kam.

Um möglichst zuverlässige Werte zu gewinnen, wurde für jede Latenzzeit eine Doppelbestimmung, gemacht und der Mittelwert eingesetzt. Dies Verfahren ermöglichte zugleich eine Berechnung der Fehlerbreite. Unter 90 Doppelbestimmungen ergab sich im Durchschnitt eine Differenz der zusammengehörigen Werte von $0,12 \sigma$, was bei den im allgemeinen gemessenen Latenzzeitunterschieden für die Leitgeschwindigkeitsbestimmung ca $7,2^{\circ} ;$ ausmachen würde. In vereinzelten Fällen erreichte obige Differenz 0,25 $\sigma$. Während in all diesen Doppelbestimmungen die beiden sich entspreehenden Kurven gleiche Form und Höhe hatten, zeigten sich in 4 Fällen Ungleichheiten im Höhen- und Zeitmaß der Kurven, derart. daß zu der flacheren Kurve die längere (bis 0,55 $\sigma$ ) Latenzzeit gehörte. Solche Bestimmungen mußten natürlich als unverwertbar ausscheiden.

Die Richtung des Reizstromes wurde für alle unsere Frage angehenden Latenzbestimmungen derart gewählt, daß bei $c$ mit $\downarrow$ und bei $p$ mit $\uparrow$ Strom gereizt wurde, also jedenorts der wirksamsten Richtung, deren Überlegenheit besonders bei $c$ deutlich in der erheblich niedrigeren Reizschwelle zum Ausdruck kommt und mit Grützner ${ }^{1}$ ) auf die entsprechend gerichteten Nerveneigenströme zurückgeführt wird. In den Versuchen 11-15 aber wurde neben dem obigen Modus zu Beginn vergleichsweise auch die Latenzzeit für den umgekehrten Reizstrom, also bei $c$ für $\uparrow$ und bei $p$ für $\downarrow$ Richtung, festgestellt. Dabei ergab sich übereinstimmend, daß diese für Reizstelle $c$ bei $\uparrow$ Strom erheblich länger ist als für $\downarrow$, nämlich i. D. 5,13 $\sigma$ gegen 4,26 $\sigma$. Es kann diese Verlängerung von $0,87 \sigma$ nicht auf Rechnung der beim $\uparrow$ Strom um den Elektrodenabstand von $2,5 \mathrm{~mm}$ vergrößerten Nervenstrecke gesetzt werden, da hierfür nur eine Latenzverlängerung von $0,092 \sigma$ anzusetzen ist. Es bleibt somit ein Wert von $0,78 \sigma$, der weit außerhalb der Fehlergrenzen liegt und vielleicht erklärt werden kann durch Einflüsse des dem Reizstrom entgegenfließenden Nerveneigenstromes und evtl. besondere Einwirkungen des näheren Querschnittes auf den Erregungsausgangspunkt. Für diese Deutung spricht, daß die Latenzverlängerung größer ist und $1 \sigma$ überschreitet gerade in den Versuchen, in denen die

1) Grützner, Pflügers Arch. f. d. ges. Physiol. 28. 1882.

Pflügers Archiv. f. d. ges. Physiol. Bd. 195. 
Reizschwellenprüfung eine große Differenz der Erregbarkeit, i. D. $270 \mathrm{~mm} \mathrm{R}$. A. für $\downarrow$ gegen $180 \mathrm{~mm}$ für $\uparrow$, ergibt und in denen bei deutlich übermaximalem Reiz für $\downarrow$ die gleiche Reizintensität für $\uparrow$ eine wesentlich kleinere Aktionsstromkurve erzeugt, während andrerseits da, wo diese beiden Erscheinungen weniger ausgesprochen sind, auch die Latenzzeitverlängerung geringer ist. Mag durch die obigen Faktoren - Gegenstrom und andere Querschnittswirkungen - eine Schwächung der lokalen Wirkung des Reizstromes herbeigeführt werden, so ist betreffs der dadurch bedingten Latenzverlängerung damit nicht entschieden, wieweit diese auf einem verspäteten Aufbruch der Erregungswelle vom Entstehungsortc beruht, wie weit auf einer Dehnung der Muskellatenz (verspätete Saitenschwankung infolge teilweiser innerer Abgleichung des an sich untermaximalen Aktionsstromes in unbeteiligten Muskelelementen) und in wie weit in der erschwerten Ausmeßbarkeit der weniger steilen Kurven. Vielleicht aber steckt in dem Wert von $0,87 \sigma$ noch ein weiteres latenzverlängerndes Moment, eine elcktrotoniche Hemmung der Erregung an der Anode, falls hier die depressive Wirkung lange genug anhält. Für dieses dürften wir nach dem folgenden nur einen geringen Zeitbetrag einsetzen, kaum mehr als $0,25 \sigma$.

An der Elektrode $p$ finden wir, viel weniger ausgesprochen und nicht so konstant, das umgekehrte Verhalten, daß nämlich bei $\downarrow$ gerichtetem Reizstrom die Latenzzeit um i. D. $0,25 \sigma$ kürzer ist als bei dem im allgemeinen von uns benutzten $\uparrow$ Strom. Auch dieser Betrag ist nur zum kleinsten Teile durch die Verkürzung der Strecke um $2 \mathrm{~mm}$ zu erklären, für die leicht eine Latenzzeit von ca. 0,07 $\sigma$ berechnet werden kann. Der an dieser Stelle im Nerven meist dem $\downarrow$ Reizstrom entgegen verlaufende Eigenstrom ist schwach und von geringer Wirkung, wie sich auch aus dem kleinen Unterschied der Schwellenerregharkeit für $\uparrow$ und $\downarrow$ Reizstrom ergibt. Seine die Latenz verlängernde Wirkung mag daher nicht groß sein und leicht durch die verkürzenden Faktoren überkompensiert werden. Als solche aber kämen in Frage etwa der Wegfall verzögernder Anodenwirkung und vielleicht in Füllen naher Lagerung der Kathode an den Muskel ein Ủbergreifen von Stromschleifen bis auf diesen. Für die letzte Möglichkeit spricht die Beobachtung, daß mehrfach der Aktionsstrom bei $\downarrow$ maximalem Reiz $\mathrm{zu}$ einem größeren Saitenaussohlag führte, als bei $\uparrow$ Reizstrom, obwohl der letzte cine etwas höhere Schwellenerregbarkeit aufwies.

Es ist etwas ausführlicher auf diese Nebenbeobachtungen eingegangen, weil sie die von uns angewandte Lage der Elektrodenpole rechtfertigen sollen. Diese erweist sich demnach für Bestimmungen der Leitgeschwindigkeit am Nerven als zweckmäßig in der Art, daß die Reizstromrichtung mit der der Nerveneigenströme übereinstimmt, was besonders für das zentrale Nervenende wichtig ist und erkannt wird an der jeweils größten Schwellenerregbarkeit, und daß möglichst die Ausgangspunkte der Erregung einander zugewandt die beobachtete Nervenstrecke begrenzen, so zwar, daß Ausbreitung erregender Stromschleifen auf das Erfolgsorgan möglichst vermieden werden. 


\section{Die Versuche.}

Der Gang der Versuche geht aus dem folgenden hervor. Nachdem das frische Nervmuskelpräparat 10-20 Min. bei gleicher Temperatur, der Grundtemperatur, ungestört in der beschriebenen Versuchsanordnung gelegen hatte, wurden die Reizschwellen und die Latenzzeiten, letztere in doppelter Aufnahme, von jeder Reizstelle, $c$ und $p$, aus bestimmt (Kurven 1). Gleich darauf wurde eine Erwärmung der 3,2 cm langen Nervenstrecke in der Wärmekammer vorgenommen mit (oberhalb 28) gleichmäßigem Temperaturanstieg von $1^{\circ}$ in $1 / 2$ Min., der Temperaturgrad für den Eintritt der Wärmelähmung ${ }^{1}$ ) notiert, mehr oder weniger hoch und lange über diesen Grad hinaus weitererwärmt, bis 39 oder gar $41^{\circ}$, um möglichst starke ,Gewöhnung" zu erzielen, und dann rasch auf Zimmertemperatur abgekühlt, wobei die Muskelzuckung auf zentralen Reiz (bei c) voll wieder auftrat. Bald darauf wurde von neuem in gleichem Tempo erwärmt, um den Umfang der ,Gewöhnung" festzustellen, und unmittelbar nach Erreichung des Temperaturgrades des Leitfähigkeitsverlustes schnell wieder abgekühlt²). Nachdem nun das Präparat bei der Grundtemperatur 15-20 Min. geruht hatte, um sich völlig auf diese abkühlen zu können, wurden als Kurven II erneute Latenzzeitbestimmungen vorgenommen, welche die Leitungsverlangsamung erweisen sollten.

In den orientierenden Versuchen $1-5$ begnügten wir uns hiermit und bestimmten auch nur die Veränderungen, die die Latenzzeit eines bei $c$ gesetzten Reizes durch die Wärmebeeinflussung der unterhalb gelegenen Nervenstrecke erfährt. Es ergab sich, daß diese Zeit nach der Überer. wärmung tatsächlich erheblich verlängert ist, i. D. um $0,53 \sigma$ (Tab. I Stab k $=$ ca. 12,5\% der durchschnittlichen Latenzzeit von $4,23 \sigma \mathrm{zu}$ Beginn. Diese Verlängerung bietet wohl einen Hinweis auf das erwartete Resul-

1) Aufhören der letzten Muskelzuckung auf Reiz bei c, eigentlich nicht Beginn, sondern schon etwas vorgeschrittenes Stadium der Lähmung, in welchem das Dekrement eben so stark geworden ist, daß die Erregungswelle in der alterierten Strecke erlischt.

2) Es mag hier anmerkend erwähnt sein, daß bezüglich der Gewöhnung unsere früheren Erfahrungen, s. o., vollauf bestätigt wurden, ja daß wir diesmal in regelmäßigerer Weise noch größere Ausschläge erreichten infolge energischerer Übererwärmung bei exakter arbeitender Methodik und besserem Froschmaterial. Im Durchschnitt von 16 Versuchen erfolgte die erste Wärmelähmung bei $34,8^{\circ} \mathrm{C}$. Die zweite aber trat erst bei $37,9^{\circ}$ ein, was einen Umfang der "Gewöhnung" von $3,1^{\circ}$ bedeutet nach einef durchschnittlichen Übererwärmung von $4,8^{\circ}$ in $3^{1} / 4 \mathrm{Min}$. (Vgl. Tab. I.) Ziehen wir aber aus dieser Gesamtzahl die Versuche mit den höchsten und längstdauernden Übererwärmungen heraus, etwa Versuch 8, 10, 11, 15 und 16, so sehen wir, daß bei einer durchschnittlichen Übererwärmung von $5,5^{\circ}$ und 4 Min. Dauer eine „Gewöhnung“ um gar $4^{\circ}$ eintritt. Damit scheint allerdings das Maximum erreicht. 
610 W. Thörner : Leitungsverlangsamung und Vorringerung des Stoffumsatzes als

tat, braucht jedoch nicht unbedingt und sicher nicht in vollem Umfange auf die Wärmealteration des Nerven bezogen werden, da anzunehmen ist, daß sowohl die Latenzzeit des Nerven überhaupt und besonders am querschnittsnahen Reizpunkt wie auch vor allem die des Nervenendapparates und Muskels allein durch die natürlichen Absterbeprozesse während der Versuchsdauer allmählich größer wird.

\section{Tabelle I.}

Übersicht über die Ergebnisse aller Versuche zur Darstellung der Abhängigkeit der Gewöhnung und Leitungsverlangsamung von Tieje und Dauer der 1. Wärnelähmung.

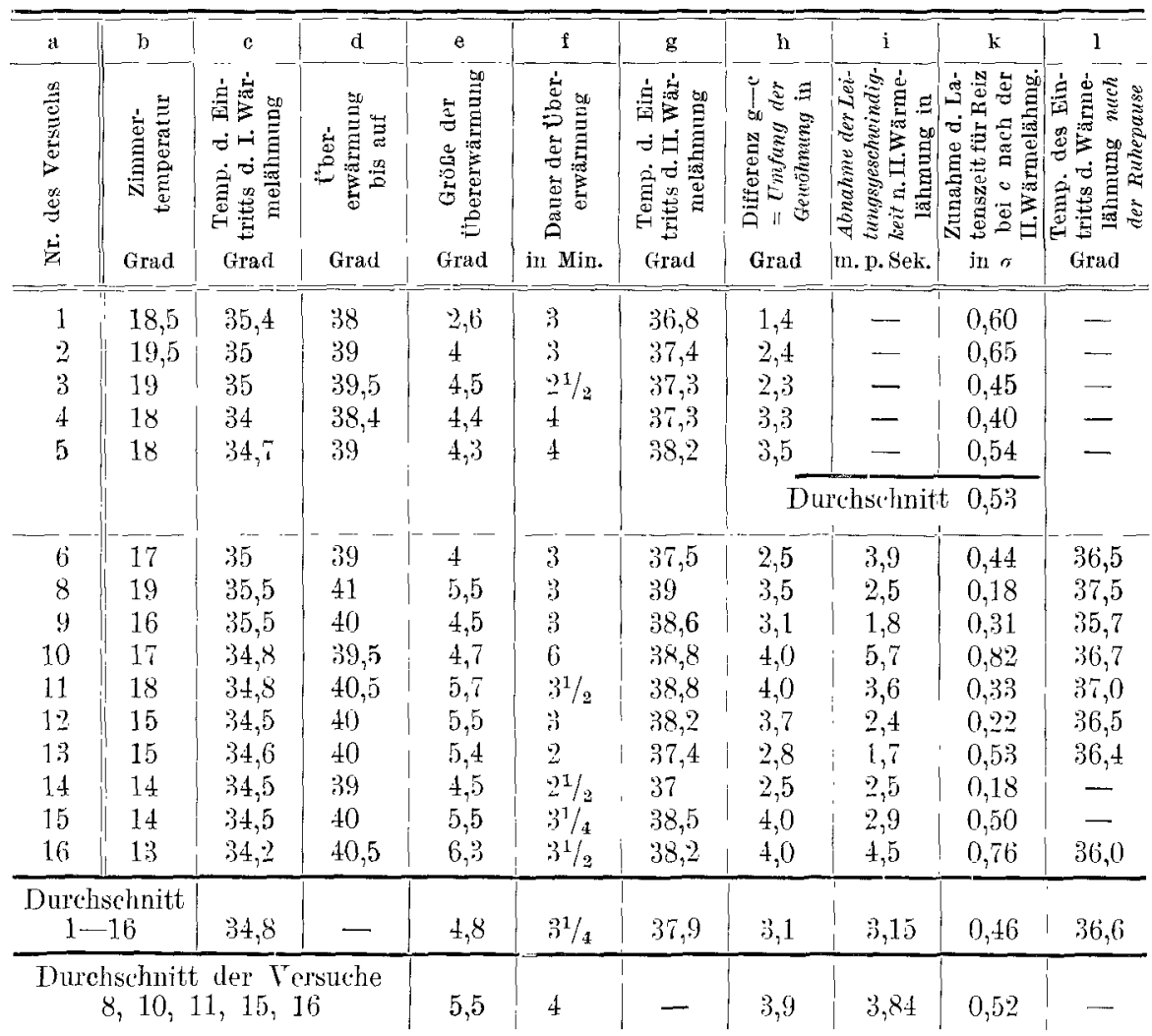

Es wurden daher in allen weiteren Versuchen, 6-16, auBerdem die Latenzzeiten für einen peripher der Wärmestrecke bei $p$ gesetzten Reiz bestimmt und damit einerseits die zwischenzeitlichen Veränderungen am peripheren Abschnitt des Präparates kontıollierbar und andrerseits eine direkte Berechnung der Leitungsgeschwindigkeit in der untersuchten Nervenstrecke möglich gemacht. 
Grundlage (l. scheinbaren „(rewöhnung" d. wärmegelähmt gewesenen Nerven. 611

T'abelle II. Zusammenstellung der an den Aktionsstromkurven ausgemessenen Werte für die Verändernngen der Latenzzeiten und daraus berechneten Leitungsgeschwindigkeiten der einzelnen Versuche unter dem Einfluß der Wärmelähmungen und der mehr-stündigen Ruhepausen.

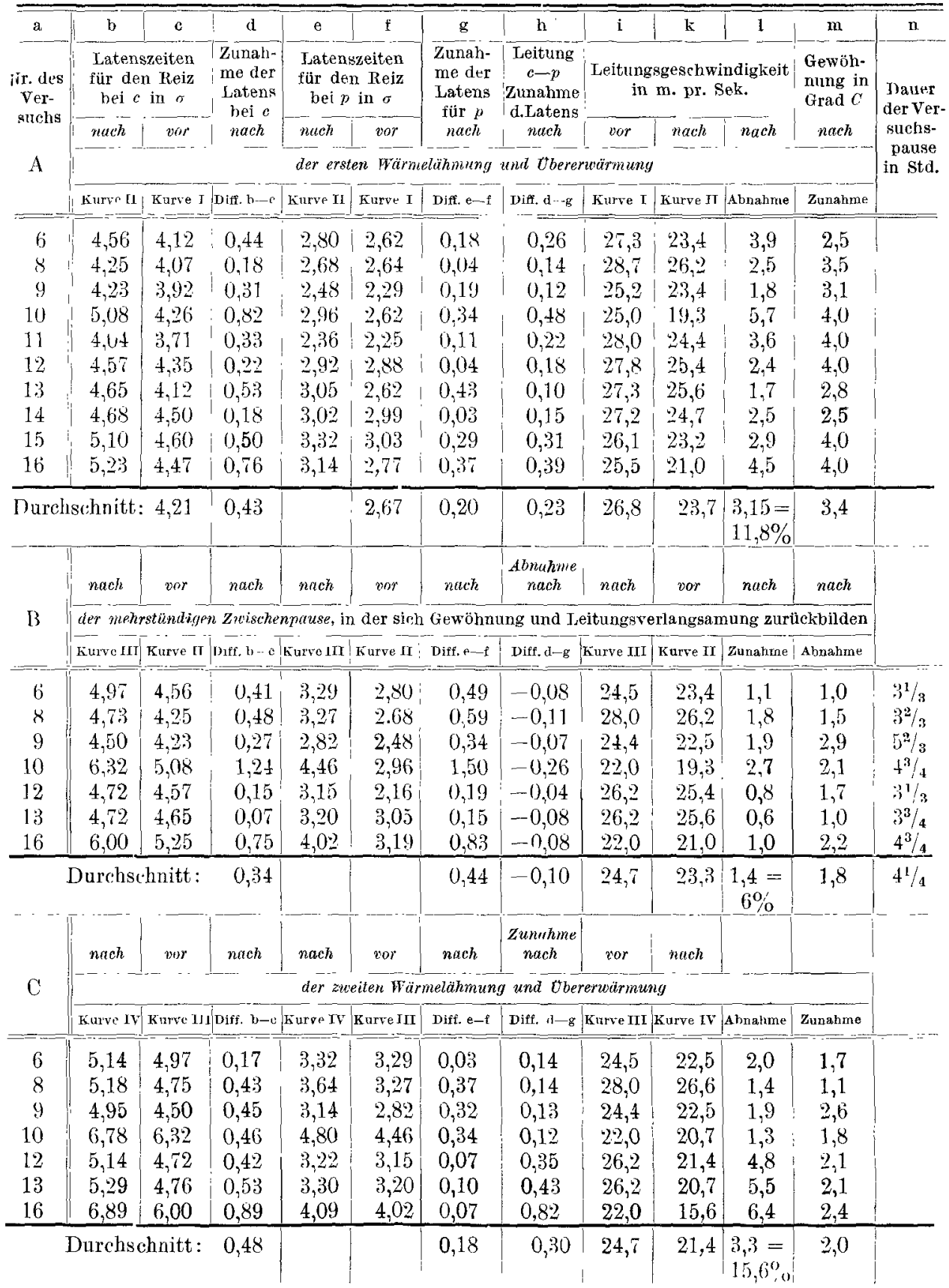


Versuchsprotokoll 12.

\begin{tabular}{|c|c|c|c|c|c|c|c|c|c|}
\hline \multirow[b]{2}{*}{ Zeit } & \multirow{2}{*}{ 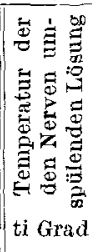 } & \multirow{2}{*}{ 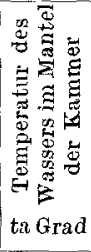 } & \multirow{2}{*}{ 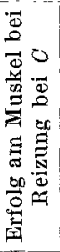 } & \multirow{2}{*}{ 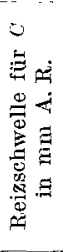 } & \multirow{2}{*}{ 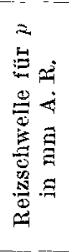 } & \multicolumn{2}{|c|}{ Latenszeit für } & \multirow{2}{*}{ 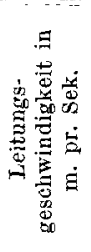 } & \multirow[b]{2}{*}{ Bemerkungen } \\
\hline & & & & & & $\begin{array}{l}\text { Reiz } \\
\text { bei } c \\
\text { in } \sigma\end{array}$ & $\begin{array}{l}\text { Reiz } \\
\text { bei } p \\
\text { in } r\end{array}$ & & \\
\hline $11^{\text {th }} 10^{\prime}$ & 14,5 & 14 & + & 293 & 190 & & & & ¿ 200 K. A. \\
\hline $11^{\mathrm{h}} 40^{\prime}$ & 15 & 14,5 & + & 275 & 180 & & & & p. $160 \mathrm{R} . \mathrm{A}$. \\
\hline $11^{\mathrm{h}} 45^{\prime}$ & 15 & 14,5 & + & 275 & 180 & 4,35 & 2,88 & 27,8 & Kurven I \\
\hline $11^{\mathrm{h}} 53^{\prime}$ & 30 & .30 & + & 280 & & & & & \\
\hline $11^{\mathrm{h}} 55^{1 / 2}$ & 34,5 & 34,8 & $\cdots$ & & & & & & $\begin{array}{l}\text { Eintritt der } \\
\text { W:irmelithmung }\end{array}$ \\
\hline $11^{\text {h }} 5 \tau^{\prime}$ & 40 & 40,5 & - & & & & & & \\
\hline $11^{\mathrm{h}} 5 \mathrm{R}^{1} / \mathrm{g}^{\prime}$ & 36,8 & $35, i$ & + & & & & & & Leitungs- \\
\hline $11^{\text {h }} 59^{\prime}$ & 25 & 15 & + & & & & & & rückkehr \\
\hline $12^{\mathrm{n}} 5^{\prime}$ & 16 & 15 & + & & & & & & \\
\hline $12^{\mathrm{h}} 11^{\prime}$ & 33 & 31 & + & & & & & & \\
\hline $12^{\mathrm{n}} 14^{\prime}$ & 38,2 & 38,2 & - & & & & & & $\begin{array}{c}\text { Eintritt der } \\
\text { Wärmelähmung }\end{array}$ \\
\hline $12^{\mathrm{h}} 15^{\prime}$ & 23 & 14 & + & & & & & & \\
\hline $12^{\mathrm{n}} 25^{\prime}$ & 15 & 14,5 & + & 285 & 180 & 4,57 & 2,96 & 25,4 & Kurven II \\
\hline $3^{\text {h }} 40^{\prime}$ & 15 & 14,2 & + & 282 & 190 & 4,72 & 3,15 & 26,2 & Kurven III \\
\hline $3^{\text {h }} 50^{\prime}$ & 30 & 30 & + & & & & & & \\
\hline $3^{\mathrm{n}} 51^{\prime}$ & 33 & 34 & + & & & & & & \\
\hline $3 n 53^{1} / 2$ & 36,5 & 36,5 & - & & & & & & $\begin{array}{c}\text { Eintritt der } \\
\text { Wïmolühmung }\end{array}$ \\
\hline $3^{h} 55^{\prime}$ & 40 & 41 & - & & & & & & \\
\hline $3^{\mathrm{h}} 57^{\prime}$ & 25 & 14 & + & 280 & & & & & \\
\hline $4^{n} 20^{\prime}$ & 15 & 14,6 & + & 270 & 190 & 5,14 & 3,22 & 21,4 & Kurven IV \\
\hline$-4 \mathrm{n} 30^{\prime}$ & 30 & 31 & + & & & & & & \\
\hline $4^{\text {h }} 33^{\prime}$ & 33 & 34 & + & & & & & & \\
\hline $4^{h} 36^{\prime}$ & 37,5 & 35,5 & - & & & & & & $\begin{array}{c}\text { Eintritt der } \\
\text { W:irmelähmmng }\end{array}$ \\
\hline $4^{\text {h }} 38^{\prime}$ & 20 & 15 & + & 280 & 185 & & & & \\
\hline
\end{tabular}

In Bestätigung der obigen Überlegung zeigte sich denn auch, daß nach der Wärmelähmung auch die von dieser nicht betroffenen peripheren Teile abwärts $p$ ein verlängertes Latenzstadium aufweisen, und zwar verlängert durchschnittlich um $0,20 \sigma=4,7 \%$ der anfänglichen Latenzzeit des Gesamtpräparates 4,21 +0,7 $\sigma$ (vgl. Tabelle II A.). Dessen Latenzzeit, Reizpunkt $c$, ist dagegen in diesen Versuchen um $0,43 \sigma=10 \%$ gewachsen, so daß sich ein Überschuß von $0,23 \sigma=5,3 \%$ ergibt und wir somit gut die Hälfte der am ganzen Präparat gefundenen Latenzzeitäunahme für Reizpunkt c auf Rechnung der beeinflußten Nervenstrecke c $p$ setzen dürfen. Die in dieser durch die Übererwärmung bedingt und riüückgelassene I eitungsterlangsamung ist es, die neben 
einer gewissen Absterbequote in der Differenz der Latenzzeitenzunahme (Tab. II A, Stab h) zahlenmäßig zum Ausdruck kommt. Den Betrag der Absterbequote kennen wir nicht, er kann aber nach Maßgabe desjenigen von Endorgan und Muskel und nach Ausweis einer Wiederkehr größerer Leitungsgeschwindigkeit, wovon noch zu sprechen, nicht erheblich sein.

Noch übersichtlicher erscheinen die Ergebnisse, wenn wir aus den gewonnenen Latenzdaten die Geschwindigkeiten der Nervenleitung für die bewußte Strecke berechnen (Tab. II A, Stab i, k, l). Betrug diese für den frischen Nerven im Durchschnitt $26,8 \mathrm{~m}$ p. S., so durchläuft die Erregung die gewöhnte Strecke nach der Übererwärmung nur noch mit $23,7 \mathrm{~m}$ p. S., also mit einer Verlangsamung von $3,15 \mathrm{~m}$ p. $\$$., d. h. um 11,8\% des Anfangswertes. Um dieses arithmetische Mittel schwankt der Betrag dieser Verlangsamung in den einzelnen Versuchen, während zwischen den Grenzfällen von 6,1\% und 22,8\% der Dichtemittelpunkt etwa bei $10 \%$ liegt. Diese Schwankungen gehen über den Bereich der Bestimmungsfehler hinaus, in ihnen macht sich viclmehr die verschiedene Tiefe der Wärmelähmung geltend. In der Tab. I läßt ein Vergleich der Stäbe $\mathrm{h}, \mathrm{i}$, e und $\mathbf{f}$ erkenmen, daß im allgemeinen die Werte für den Umfang der ,Gewöhnung" und für die Größe der Leitungsverlangsamung parallel gehen und in gleichem Sinne abhängen von der Stärke der voraufgegangenen Übererwärmung. Während im Gesamtdurchschnitt die "Gewöhnung" 3,1 and die Leitungsverlangsamung $3,15 \mathrm{~m}$ p.S. bei einer Übererwärmung in Höhe von $4,8^{\circ}$ und $3^{1 / 4}$ Min. Dauer beträgt, ergibt eine Zusammenstellung der Versuche mit stärkerer Übererwärmung $(8,10,11,15,16)$ um $5,5^{\circ}$ in 4 Min. neben einer ,Gewöhnung" um 3,9 auch eine Abnahme der Leitgeschwindigkeit um 3,84 $\mathrm{m}$ pr. $\mathrm{s}$.

Nachdem insoweit die Versuche übereinstimmend das Ergebnis geliefert hatten, daß unter den gleichen Bedingungen wie die ,Gewöhnung" auch eine Verlangsamung der Erregungsleitung sich während der Wärmelähmung entwickelt und nachher zurückbleibt, wäre der Finwand zu berücksichtigen, die beobachtete Leitungsverlangsamung könne zum größeren Teil durch natürliches, vielleicht durch die Erwärmung beschleunigtes, Absterben der Nervenstrecke $c p$ bedingt sein. Dieser Einwand würde aber auf keine Weise besser widerlegt werden als durch den Nachweis der Fähigkeit dieser Strecke zu einer erneuten Zunahme der Leitungsgeschwindigkeit, wie sie nach den obigen (S. 604) Ausführungen zugleich mit dem Rückgang der "Gewöhnung" zu erwarten wäre, wenn man das Präparat mehrere Stunden unberührt bei Zimmertemperatur ruhen ließe. Ein positiver Ausfall derartiger Versuche würde zudem eine neue Stütze für die gemutmaßten Zusammenhänge abgeben. Und so wurden denn in 7 Fällen $(6,8,9,10,12,13,16$,) die Versuche dementsprechend fortgesetzt und führten zu Resultaten, 
wie sie in Tab. II unter $\mathrm{B}$ und $\mathrm{C}$ zusammengestellt sind. Als Beispiel diene auch Protokoll 12. Nach dem ersten Teil des Versuches, an dessen SchluB die Latenzzeiten als Kurvenserie II 10-20 Min. nach Abfall der Erwärmung bestimmt waren und die Leitungsverlangsamung ergeben hatten, blieben die Präparate vor allen äußeren Schädigungen möglichst geschützt in der Versuchsanordnung bei Zimmertemperatur 3-6 Stunden liegen. Diese Ruhepause (Stab $n$ ) länger auszudehnen, wie vielleicht zur vollständigeren Reduktion der Gewöhnungsbedingungen wünschenswert gewesen wäre, ging nicht an, da die Aktionsströme des Muskels mit der Versuchsdauer immer kleiner wurden, während doch ihre Größe im Interesse einer präzisen Kurvenwertung nicht unter ein gewisses Maß absinken durfte. - Der Nerv allein hätte wohl länger ausgehalten, wenigstens zeigte seine Erregbarkeit (Prot. 12) auch nach vielstündigen Versuchen kaum eine Einbuße. Aber Nervenaktionsströme lieferten uns für den Zweck zuverlässiger Kurvenmessung zu kleine Saitenausschläge. - Die Versuchspause in den angebenen Grenzen genügte aber vollkommen, um neben einem deutlichen Rückgang der Gewöhnungserscheinungen eine einwandfreie Zunahme der Leitungsgeschwindigknit zustande zu bringen.

In der Ruhepause von i. D. $4^{1} / 4$ Stunde haben die Latenzzeiten infolge der Absterbeprozesse natürlich weiter zugenommen, aber im Gegensatz zu früher jetzt die für den Reizpunkt $c$ weniger als für $p$ (Tab. II B, Stab d, g, h); das spricht unbedingt für eine relative Erholung der Zwischenstrecke $c p$, derart, daß die Erregung, um diese zu passieren, jetzt ca. $0,10 \sigma$ weniger gebraucht als vor der Pause. Noch anschaulicher läBt sich das durch Berechnung der Leitungsgeschwindigkeiten darstellen, wie sie in Tab. II B, Stab i als Kurve III notiert sind. Diese zeigen, verglichen mit den Werten von Kurve II, Stab $\mathrm{k}$, vor der Pause eine Zunahme i. D. um 1, $4 \mathrm{~m}$ p.S. $=6 \%$ früheren Wertes, womit demnach trotz der fortschreitenden Absterbeprozesse fast die Hälfte des durch dio Übererwärmung bedingt gewesenen Leitgeschwindigkeitsverlustes wieder eingeholt ist. - Es geht aus diesen letzten Bestimmungen übrigens hervor, daß die Absterbevorgänge eine Verlangsamung der Erregungsund Leitungsabläufe vor allem wohl im Endorgan und Muskel hervorrufen und viel weniger die Nervenfaser treffen, zumal wir, wenn wir von den willkürlichen Beeinflussungen der Strecke $c p$ einmal Abstand nehmen, die Latenzzeiten für $c$ und $p$ fast gleichmäBig zunehmen sehen und nicht etwa wesentlich schneller für $c$, wie den Längenverhältnissen nach zu erwarten wäre. - Im einzelnen schwankt die Größe der Zunahme der Leitgeschwindigkeit und wir bemerken, daß sie im allgemeinen um so beträchtlicher ist, je länger die Ruhepause gedauert hat (vgl. Stab $1, \mathrm{~m}, \mathrm{n}$ ). Während der Gewinn in Versuch 6, 8, 12, 13 nach ca. $33_{4}$ Stunde Pạke bei $1,3^{\circ}$ Gewöhnungsrückgang nur $1,1 \mathrm{~m}$ p. S. $=4,4^{\circ}$ 
beträgt, steigt er in den drei übrigen Versuchen mit ca. 5 Stunden Pause neben einem Rückgang der Gewöhnung um $2,4^{\circ}$ auf $1,9 \mathrm{~m} \mathrm{p}$. S. $=8,3 \%$.

An die Latenzzeitbestimmungen, die eine derartige Wiederkehr höherer Leitungsgeschwindigkeit nach der mehrstündigen Pause ergeben hatten, wurde in den genannten Versuchen eine abermalige Erwärmung angeschlossen, welche die durch die Versuchspause herbeigeführte Rückbildung der ,Gewöhnung“ zum Ausdruck brachte. War vor der Pause die Wärmelähmung zuletzt hei einer erhöhten Temperatur von durchschnittlich $38,2^{\circ}$ eingetreten, so erfolgte sie jetzt wieder bei niedriger Temperatur, i. D. bei $36,4^{\circ}$ (Tab. II, Stab 1), was einen Verlust der anfangs gewonnenen ,Gewöhnung" um 1,8 ${ }^{\circ}$, d. h. um etwa die Hälfte des ursprünglichen Betrages (vgl. Tab. II B, Stab m, n, l) entsprechen und sehr gut zu der ebenfalls um fast die Hälfte des Verlorenen gesteigerten Leitungsgeschwindigkeit passen würde. Ja, in dem Versuche 9 mit der längsten Ruhepause von $5 \% / 3$ Stunde ist die ,Gewöhnung" fast ganz zurückgegangen und die letzte Wärmelähmung nahezu wieder bei der Temperatur der ersten eingetreten; hier finden wir gleichzeitig einen ziemlich beträchtlichen Zuwachs der Leitgeschwindigkeit.

Eine über diese letzte Wärmelähmung hinaus forgeführte Übererwärmung hatte naturgemäß von neuem ,Gewöhnung" zur Folge. Nur konnten deren Beträge, i. D. $2^{\circ}$, nicht so groß sein wie an frischen Präparaten, da sie sich auf die durch nicht völligen Rückgang der ersten Gewöhnung erhöht gebliebenen Wärmelähmungstemperaturen aufsetzten und daher der Spielraum bis zur Todestemperatur eingeengt war. Gleichzeitig sehen wir aber eine kräftige Leitungsverlangsamung in die Erscheinung treten, die die ersterzengte vielleicht an Umfang noch übertrifft (vgl. Tab. II C). Sie beträgt i. D. $3,3 \mathrm{~m} \mathrm{p.S.}=15,6 \%$. Es scheint, daß der lange im Versuch befindliche Nerv mit dem Fortschreiten der natürlichen Absterbevorgänge empfindlicher gegen die Wärmoschädigungen wird, indem diese umfangreicher und mehr und mehr irreversibel werden (Gerinnung). Ein solcher Nerv würde also durch erneute Wärmelähmung beschleunigt dem Tode entgegengeführt werden und wohl nicht mehr imstande sein, durch längere Ruhe bei Zimmertem. peratur einen beträchtlicheren Teil der verlorenen Leitungsgeschwindigkeit wiederzugewinnen.

Überblicken wir die Versuche, so kann als deren wesentliches Ergebnis folgendes als festgestellt gelten: Nach einer über den Eintrittspunkt der Wärmelähmung um einige Grade fortgesetzten Erwärmung ( $\ddot{U}$ bererwärmung) bleibt der Nerv nach Abkühlung in einem Zustand zurück, in dem er einer erneuten Erwärmung mit größerem Widerstand begegnen kann (Eintritt der zweiten Wärmelähmung bei um 3-1 1 $^{\circ}$ herer Temperatur). Dies Verhalten wurde als „Gewöhnung" bezeichnet. Der gewöhnte 
616 W. Thörner: Leitungsverlangsamung u. Terringcrung des Stoffunsatzes usw.

Nerv erweist sich ebenfalls als widerstandsfähiger gegen direkte Sauerstoffentziehung (um 8\%, verzögerte Erstickung in reinem Stickstoff). Gleichzeitig mit der Gewöhnung zeigt ein solcher Nerv eine Verlangsamung der Leitungsgeschwindigkeit um 3,15 m p. S. $=11,8 \%$. Gewöhnung und Leitungsverlangsamung sind in gleichem Sinne abhängig von der Intensität der voraufgegangenen Übereruärmung. Beide bilden sich um gut die Hälfte ihres Betrayes zurück, wenn der Nerv mehrere Stunden bei Zimmertemperatur ruht.

Diese Ergebnisse stützen die Annahme, daß die Gewöhnungserscheinungen auf eine Stoffwechselverlangsamung zurückzuführen sind, die den Sauerstoffbedarf einschränkt. Diese Stoffwechselverlangsamung muß reversibel sein, da mit den Gewöhnungserscheinungen auch die Leitungsverlangsamung zurückgebildet werden kann. Es müssen ihr also reversible Vorgänge im Protoplasma zugrunde liegen. Welcher Art. diese sein können, läßt sich nicht näher bestimmen. Wahrscheinlich handelt es sich um reversible Zustandsänderungen der Protoplasmakolloide (Gerinnungen). Unsere Anschauungen darüber sind in der Eipleitung ausführlicher dargestellt. 